\title{
Urban Landscape Information Atlas and Model System Based on Remote Sensing Images
}

\author{
Tian $\mathrm{Hu}^{1}$ and Wenbin Gong $\mathbb{1 D}^{2}$ \\ ${ }^{1} X i$ 'an University of Science and Technology, College of Architecture and Civil Engineering, Xi'an 710054, Shaanxi, China \\ ${ }^{2}$ School of Architecture, Southwest Minzu University, Chengdu 610041, Sichuan, China \\ Correspondence should be addressed to Wenbin Gong; 21900064@swun.edu.cn
}

Received 17 May 2021; Revised 4 June 2021; Accepted 23 June 2021; Published 3 July 2021

Academic Editor: Sang-Bing Tsai

Copyright ( $) 2021$ Tian Hu and Wenbin Gong. This is an open access article distributed under the Creative Commons Attribution License, which permits unrestricted use, distribution, and reproduction in any medium, provided the original work is properly cited.

\begin{abstract}
City landscape is an element of city image, and city landscape logo should also belong to the entire system of the city image logo. Since the reform and opening up, China's economic construction industry has attracted worldwide attention, and the improvement of people's living standards has led to the continuous development of urban construction. The original urban style is gradually replaced by high-rise buildings, the differences between cities are getting smaller and smaller, and the people living in them have gradually abandoned the inheritance of various cultures and customs. This paper aims to study the urban landscape information map and its model system based on remote sensing images. With the support of remote sensing image technology and geographic information system platform, the urban landscape information map model system is developed, which can vividly reflect the changes in the urban landscape pattern. The urban landscape information map is used to display and reveal the spatial evolution of the urban landscape in the process of urban development. Result of empirical analysis: it summarizes the methods of geoscience information map and urban landscape information map and establishes the goal and content of researching urban landscape information map. And it provides a basis for solving the problems of urban development and urban management. In 2021, Shanghai's urban areas have occupied more than $80 \%$. It is conceivable that the area of arable land must be very small, but the area of green land still accounts for $40 \%$; compared with last year, it is still an increase of $6 \%$.
\end{abstract}

\section{Introduction}

1.1. Background of Topic Selection. With the development of social economy, the process of urbanization is getting faster and faster. As China adopts urban management and county-level management methods to promote economic development in rural areas, urban expansion has had a relatively large impact on urban construction areas, urban and rural suburbs, and rural residential areas. Natural landscapes such as forests, grasslands, and water bodies in the enclosed area are transformed into urban landscapes such as agricultural land, residential land, industrial land, and mining land. With the influence of China's reform and opening up policy, many new cities have appeared, and the old cities have also undergone tremendous changes. As a result of these changes, the spatial structure of the urban landscape will also change. Urbanization is an inevitable trend of social development and will inevitably bring about a series of ecological problems. Therefore, it is necessary to study the changes of the urban landscape information map and its model system.

1.2. Significance of the Research. The design of many city squares in our country emphasizes the same large-scale and three-dimensional space and has certain defects in originality, innovation, and aesthetics. The point of view of this research has been fixed in the "urban landscape information map and its model system," and it has left a deep impression on how to use graphics that directly express the uniqueness of the square in the urban landscape. It made a meaningful attempt to provide a practical reference value for practice. 
The relevant research on the urban landscape information map and its model system ecological planning is helpful to solve the urban ecological crisis, and the construction of a good, harmonious, and sustainable urban environment is of great significance to all aspects of urban development. Remote sensing images have the advantages of powerful macroscopic observation capabilities, obvious benefits of dynamic monitoring, multiple detection methods, large amounts of data, and comprehensive and comparable data. They can be used to study urban landscape information maps and their model systems.

\subsection{Related Work of Urban Landscape Information Map and} Its Model System Research. Gong C recommends using landscape accessibility as an indicator to evaluate the service function of the urban green space system to citizens and emphasizes the importance of the spatial distribution pattern of green space [1]. However, I think that due to the limitation of disciplines, the lack of research on remote sensing image technology will inevitably lead to the inability to understand the fundamental reason of the urban landscape information map and its model system. Silvia V suggested that, through research on the "Urban Landscape Information Atlas and Model System" of the United States, we should formulate a strategic deployment for the longterm urban landscape, integrate the ecological, entertainment, and cultural functions of the greenway network, and strengthen the map at all levels [2]. Huang et al. summarized the development trend of the international urban landscape information map and its model system and concluded that the modern urban landscape has ecological, entertainment, natural and cultural heritage values [3]. However, I think that, due to the limitation of disciplines, the lack of research on remote sensing image technology will inevitably lead to the inability to understand the fundamental reason of the urban landscape information map and its model system.

\subsection{Innovation Points of This Research}

(1) The remote sensing image method is used to study the urban landscape information map and its model system, which makes the research of the urban landscape information map and its model system more convenient [4]

(2) Remote sensing images have the advantages of strong macroscopic observation capabilities, obvious benefits of dynamic monitoring, multiple detection methods, large amounts of data, and comprehensive and comparable data, which can be a good study of urban landscape information maps and their model systems [5]

\section{Urban Landscape Information Map and Its Model System Research Method}

\subsection{Perspectives on Urban Landscape Research}

2.1.1. Landscape Ecology. It is a trend to use landscape ecology to study the urban landscape, and it is also the main method to study the urban landscape. Landscape ecology is constantly developing. Landscape ecology emphasizes the maintenance and development of the heterogeneity of urban landscapes and the interaction between ecosystems. In the process of development, it continuously absorbs the methods of geosciences, ecosystems, system theory, and other disciplines. Therefore, it has been widely used in the study of urban landscape [6].

2.1.2. Urban Environment Analysis. Urban environment analysis is to rationally plan the construction of urban landscapes and is an important foundation for harmonious coexistence between humans and the environment [7].

The perspective of urban landscape research is shown in Figure 1.

(1) Urban Planning. Urban planning emphasizes the characteristics of the city, so the design of the urban landscape must meet the aesthetic requirements of humans [1]. Urban planning aims to promote the harmonious development of man and nature by rationally arranging various elements of urban space.

(2) Geoscience Information Atlas. Geoinformation Atlas is a new method to study the urban landscape, and the geoinformation Atlas is based on the application of geoinformation science and remote sensing [4]. The use of geoscience information map to study the urban landscape can describe the past, present, and future of the urban landscape and discover the evolution process of the urban landscape [8].

\subsection{Classification of Urban Landscape Information Atlas.} The urban landscape information map can be divided into research objects, which can be divided into a single element map of the urban landscape and a comprehensive element map of the urban landscape. At the same time, it can be divided into an indication map, a diagnosis map, and a decision map according to the map method and function [9].

The classification of the urban landscape information map is shown in Figure 2.

2.3. Elements of Landscape Design. As far as the current economic development is concerned, the development of traditional industries has matured and improved, and only emerging industries can make new economic breakthroughs. The urban square landscape space is composed of five landscape design elements: topography, plants, paving, water, and buildings. These five landscape design elements can be reasonably combined to form a landscape space with good landscape effects [10].

2.3.1. Topographic Elements. The topographic elements of landscape design refer to the artificial natural topography and geometric topography designed according to the needs of the space function and the original topography of the site. They are the framework and other elements of the space design. It is also the basis of other landscape designs [11]. 


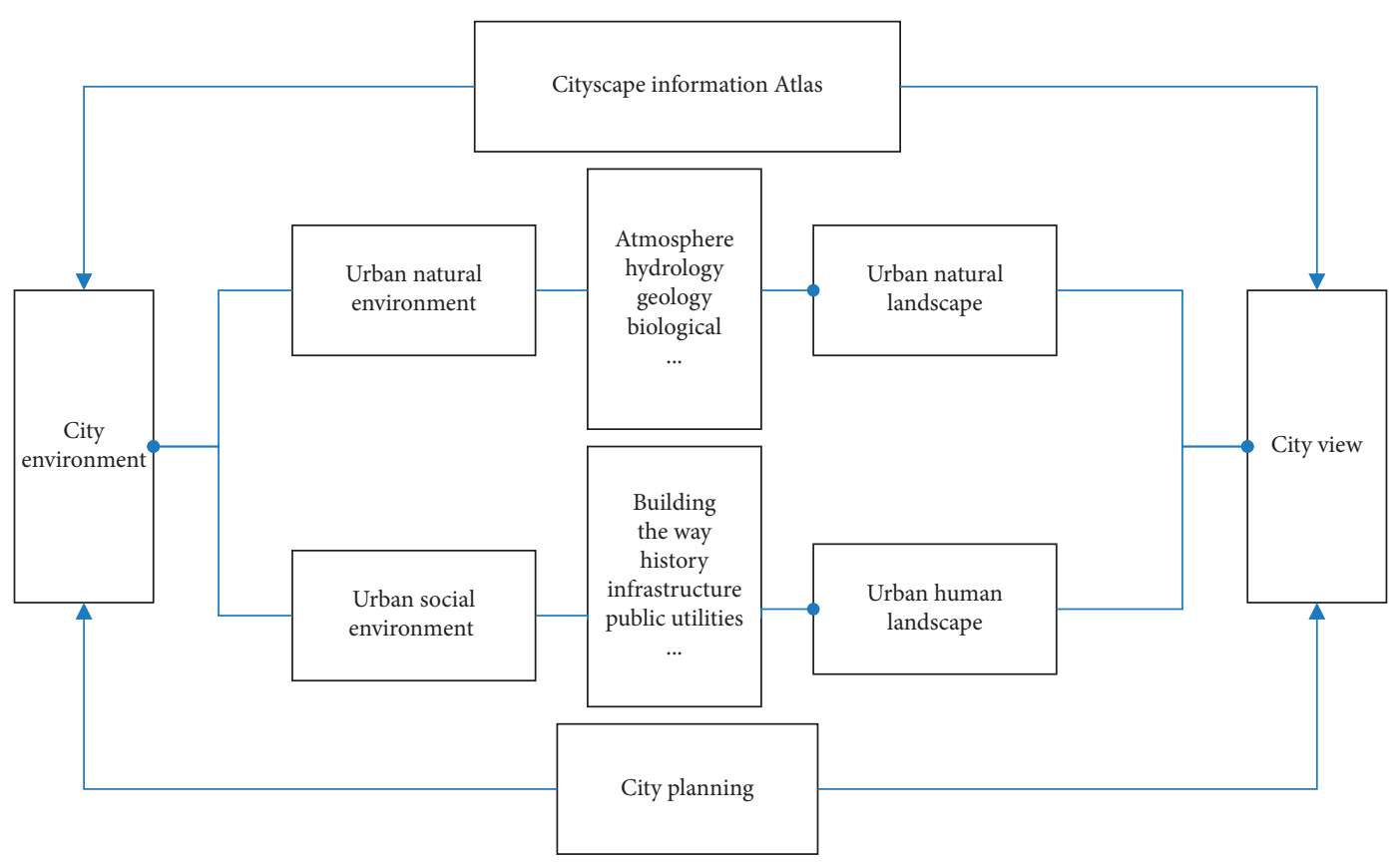

FIgURE 1: Urban landscape research perspective.

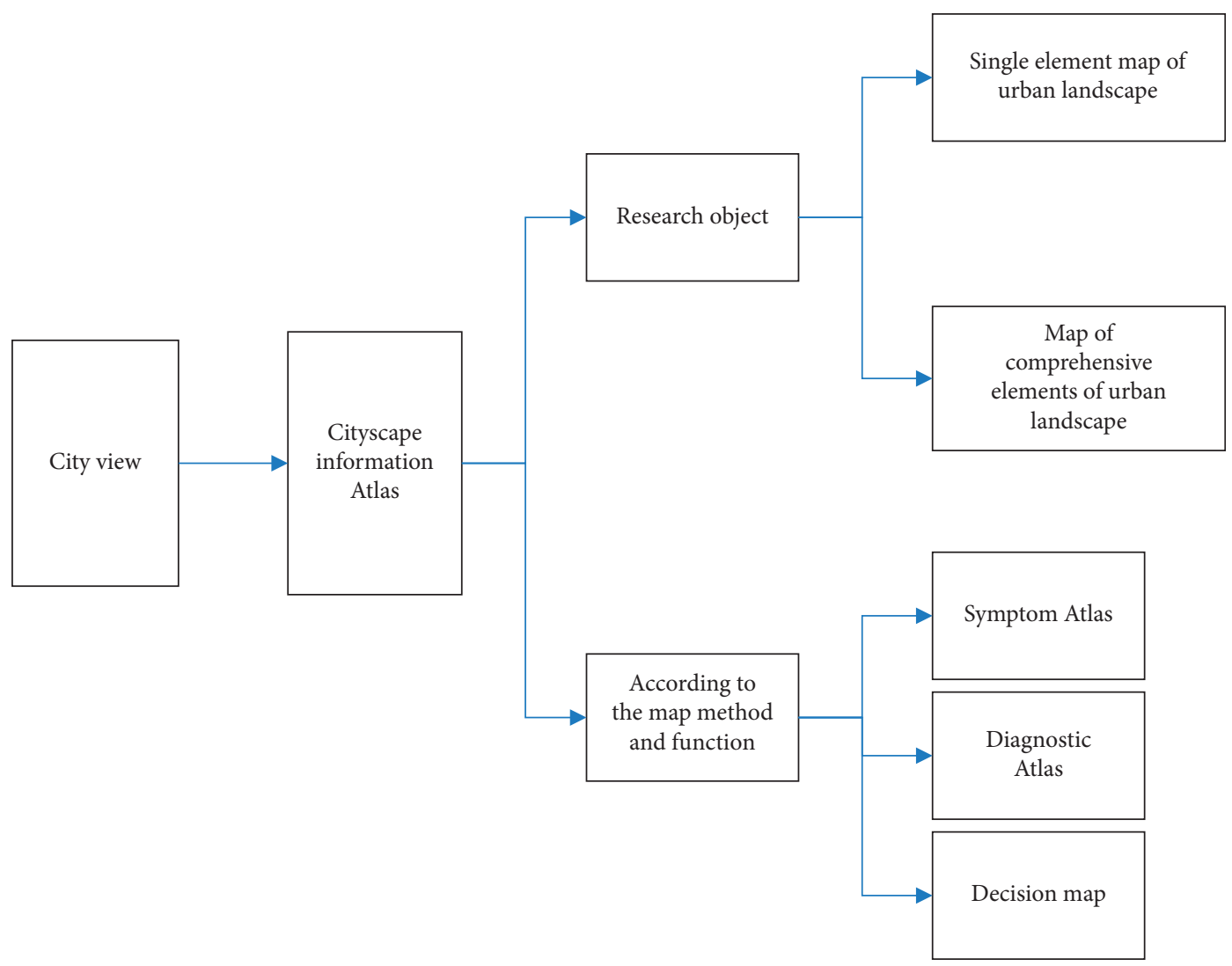

Figure 2: Classification of urban landscape information Atlas.

2.3.2. Plant Elements. Plants are the only residential design element in urban landscape design. Plants can be combined to show the seasonal changes of the space, and it can also create a better space [12].
2.3.3. Paving Elements. The paving element is used as a carrier in the urban landscape to convey people's behavior. Different packaging styles will bring different psychological emotions to people, and different styles and textures will 
have different psychological effects on people [13]. It can be seen that paving can improve the square environment, and various paving styles can be used to create various spatial atmospheres.

2.3.4. Water Element. In urban landscape design, the water body is designed according to space requirements. The water body can be designed as dynamic water, falling water, fountains, and so forth, or the water can be designed as a static form, or a combination of dynamic and static waterscapes. The water body has formability and can play a regulating role in the entire landscape space of the square [14].

2.3.5. Building Elements. In a complete urban landscape space, topography, plants, sidewalks, and other elements cannot fully meet the space requirements, and landscape structure design is also required [15]. For example, auxiliary facilities such as landscape sculptures, leisure seats, and lighting facilities can not only provide functions but also decorate and beautify the landscape space. Leisure seats provide a relaxing space for the space, and lighting facilities are especially important in the space [16]. The lighting function also determines the success of the night scene.

2.4. Characteristic Level of Urban Landscape Pattern. As China adopts urban management and county-level management methods to promote economic development in rural areas, urban expansion has had a relatively large impact on urban construction areas, urban and rural suburbs, and rural residential areas city and country development. Natural landscapes such as forests, grasslands, and water bodies in the enclosed area are transformed into urban landscapes such as agricultural land, residential land, industrial land, and mining land. We divide the urban landscape index into patch level index, patch type level index, and landscape level index [17].

The specific situation is shown in Table 1 .

\section{Experiments on the Research of Urban Landscape Information Map and Its Model System}

\begin{abstract}
3.1. Planning Steps of Urban Landscape Information Map System. The design of the urban landscape logo should integrate all aspects of the city, and it is a systematic design centered on the overall image of the city. Under the guidance of landscape ecology and information map system, a detailed study of the urban landscape planning process is carried out. The urban landscape logo can be used as a guiding system element serving the urban tourism industry, as well as an auxiliary element of the city logo, belonging to a large-scale urban image system. The steps of urban landscape ecological planning are as follows.
\end{abstract}

3.1.1. Establishing Development Direction. Each city has its own characteristics and cultural background, and each place has a different local spirit. Before carrying out the urban landscape ecological planning, it is necessary to investigate and understand the urban background and finally determine the urban development direction that is compatible with the current situation of the urban landscape [18].

3.1.2. Collecting Basic Information. After determining the development direction, a detailed investigation should be made on the city's natural resources, humanities, and land use conditions, and the corresponding basic data should be collected [19]. Due to the nature of urban landscape ecological planning, attention should be paid to the collection and classification of urban landscape data and the consideration of corresponding ecological influence factors in the data collection process [20].

3.1.3. Suitability Analysis. It organizes all the basic data collected, classifies and summarizes urban landscapes according to various adaptabilities, and especially analyzes the ecological adaptability of various landscapes.

3.1.4. Overall Planning of Ecological Green Space System. When planning the surrounding construction sites, it is necessary to distinguish the development law of various ecological elements in the urban planning area and explore the relationship between urban form and structure and the development direction of these ecological elements [21]. From the perspective of landscape ecology and ecological planning and design, it studies the overall characteristics of the urban landscape structure layout and establishes the future planning and construction direction of the urban landscape information map and its model system.

3.1.5. Developing Subplans for the Urban Landscape Information Map and Its Model System. According to the determined overall direction of urban landscape development and the content of the overall plan, a detailed plan of the urban landscape information map and its model system is established [22]. The contents of the subplans include various types of green space plans, wetland protection plans, biodiversity plans, and species plans. This subplan should be based on landscape ecology theory and ecological planning and design, follow the principles of ecology, establish a reasonable urban landscape ecological pattern, and effectively absorb the ecological benefits of various urban landscapes [23].

\subsection{Urban Landscape Analysis Method}

(1) Shape index: $S=C / 2 \sqrt{\pi A}$, where $C$ is the perimeter of the patch, $A$ is the area of the patch, and $i$ is a constant. The shape index mainly measures the complexity of the shape of the landscape patch.

(2) Fractal dimension: $A(r)$ is the surface area, $V(r)$ is the volume, $r$ is the measurement scale, and $B$ is the fractal dimension: 
TABLE 1: Hierarchical and quantitative methods of urban landscape pattern feature analysis.

Landscape pattern level

Plaque level index

Plaque type level index

Landscape level index
Corresponding landscape index

Reflected landscape characteristics

Single patch area, shape, fractal dimension, etc.

Average patch area, average shape index

Patch density, boundary density, diversity index, uniformity index
Basic characteristics and shape complexity of landscape patches

Basic characteristics, shape complexity, and spatial autocorrelation of patch types

The number of landscape types and the uniformity of type distribution

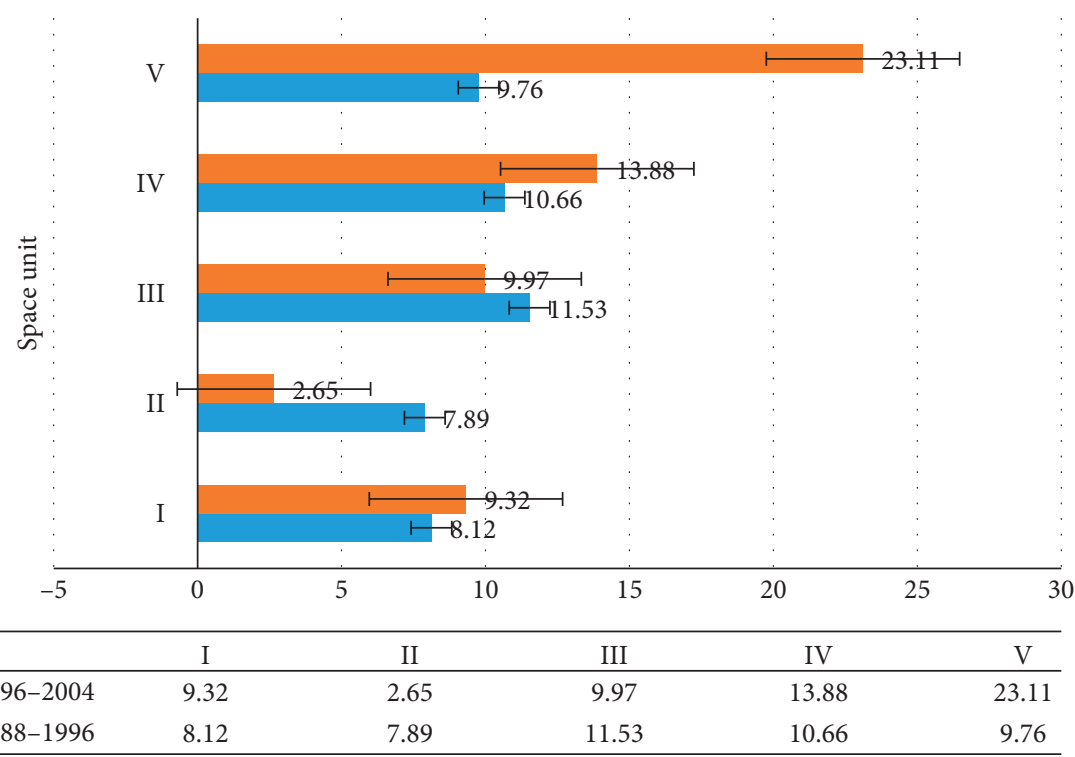

Average annual growth rate

- 1996-2004

- 1988-1996

Figure 3: The average annual expansion rate of the district (\%) from 1988 to 2004.

$$
[A(r)]^{1 / B} \sim[V(r)]^{1 / 3}
$$

The calculation formula of the fractal dimension suitable for $n$-dimensional Euclidean space is derived using the above model:

$$
[A(r)]_{n-1}^{1 / B}=i r^{\left(n-1-B_{n-1}\right) / B_{n-1}}[V(r)]^{1 / n} .
$$

If we set $n=2$ and use $r$ as the scale to measure its perimeter and area, we will get the following results: $[C(r)]^{1 / D}=i r^{(1-D) / D}[A(r)]^{1 / 2}$, where $C(r)$ is the perimeter of the landscape patch and $A(r)$ is the area. After logarithmic transformation of this formula, we get

$$
\ln (A(r))=\frac{2}{B} \ln (P(r))+C .
$$

The closer the $B$ value is to 1.5 , the more unstable the element is, and the stability index (SI) of various landscape elements can be defined as

$$
\mathrm{SI}=|1.5-B| \text {. }
$$

(3) The standard deviation of the plate area is the area of the squad block numbered $k_{j} ; A$ is the total area of the plaques, and $M$ is the total number of this type of plaques:

$$
B=\sqrt{\frac{\sum_{k=1}^{m} \sum_{j=1}^{n}\left[a_{k j}-A / M\right]^{2}}{M}} .
$$

(4) Fragmentation index: $A$ is the total area of plaques and $\sum_{i=1}^{n} M$ is the total number of all plaques:

$$
P=\frac{\left(\sum_{i=1}^{n} M\right)}{A} \text {. }
$$

(5) Uniformity index: $P_{i}, n$ has the same meaning as the calculation formula $H_{\max }=\ln (n)$ of diversity index:

$$
E=\frac{H}{H_{\max }}=\frac{-\sum_{i=1}^{n} P_{k} \ln \left(P_{k}\right)}{\ln (n)} .
$$

\section{Urban Landscape Information Map and Its Model System}

\subsection{Urbanization Issues}

4.1.1. Cultivated Land Area. With the development of cities, the area of arable land is getting less and less, the average 
area of arable land patches decreases, and the degree of separation and fragmentation increases. With the acceleration of urbanization, it is inevitable to occupy cultivated land, but it is necessary to maintain cultivated land as a social, economic, and ecological support system [24].

4.1.2. Woodland. With the acceleration of urbanization, the area of forest land is also decreasing. Most of the reason is that human cutting down trees, natural disasters, insect pests, and other natural phenomena have reduced the area of forest land. With the reduction of forest land, global warming will lead to destruction of natural ecosystems $[25,26]$.

\subsubsection{Urban Industrial, Mining, and Residential Land.} The process of urbanization has led to an increase in the area index and a gradual decrease in plate density. These all illustrate the expansion of urban industrial, mining, and residential land.

4.1.4. Water Body. The reduction of water bodies is not clear, but detailed data comparison shows that the water bodies have decreased, the patches in the water bodies have decreased, and the average patch area has decreased. The main reason is the reclamation and expansion of the entire city $[27,28]$.

4.2. Experimental Data Analysis. First, the area of 5 spatial units was counted, in 1988, 1996, and 2004. During the period 1996-2004, the growth rates of the five spatial units of I-V were $9.32 \%, 2.65 \%, 9.97 \%, 13.88 \%$, and $23.11 \%$. In the 1988-1996 period, the growth rates of the five spatial units of I-V were $8.12 \%, 7.89 \%, 11.53 \%, 10.66 \%$, and $9.76 \%$, respectively.

The average annual expansion rate of the built-up area from 1988 to 2004 is shown in Figure 3.

\section{Conclusion}

As far as the current economic development is concerned, the development of traditional industries has matured and improved, and only emerging industries can make new economic breakthroughs. Tourism and cultural industries each represent the public environment and spiritual and cultural aspects of the city and require long-term planning and policy incentives. The urban landscape logo can be used as a guiding system element serving the urban tourism industry, as well as an auxiliary element of the city logo, belonging to a large-scale urban image system. The design of the urban landscape logo should integrate all aspects of the city, and it is a systematic design centered on the overall image of the city. It summarized the methods of geoscience information map and urban landscape information map and established the goal and content of researching urban landscape information map. And it provides a basis for solving the problems of urban development and urban management.

\section{Data Availability}

The data that support the findings of this study are available from the corresponding author upon reasonable request.

\section{Conflicts of Interest}

The authors declare no potential conflicts of interest with respect to the research, authorship, and/or publication of this article.

\section{References}

[1] C. Gong, J. Han, and X. Lu, "Remote sensing image scene classification: benchmark and state of the art," Proceedings of the IEEE, vol. 105, no. 10, pp. 1865-1883, 2017.

[2] V. Silvia, M. David, I. Jordi et al., "Production of a dynamic cropland mask by processing remote sensing image series at high temporal and spatial resolutions," Remote Sensing, vol. 8, no. 1, pp. 1-21, 2016.

[3] L. Huang, C. Chen, L. Wei et al., "Remote sensing image scene classification using multi-scale completed local binary patterns and Fisher vectors," Remote Sensing, vol. 8, no. 6, pp. 1-17, 2016.

[4] N. Gürsoy and I. Kaya, "Detecting of lithological units by using terrestrial spectral data and remote sensing image," Journal of the Indian Society of Remote Sensing, vol. 45, no. 2, pp. 1-11, 2016.

[5] Z. Shao, L. Wang, Z. Wang, and J. Deng, "Remote sensing image super-resolution using sparse representation and coupled sparse autoencoder," IEEE Journal of Selected Topics in Applied Earth Observations and Remote Sensing, vol. 12, no. 8, pp. 2663-2674, 2019.

[6] S. Liu, W. Hou, and X. Zhao, "Unsupervised object segmentation of high-resolution remote sensing image based on DPMM and MRF," Yi Qi Yi Biao Xue Bao/Chinese Journal of Scientific Instrument, vol. 39, no. 11, pp. 222-231, 2018.

[7] J. Liu, X. Li, K. Zhu et al., "Distributed compressed sensing based remote sensing image fusion algorithm," Journal of Electronics \& Information Technology, vol. 39, no. 10, pp. 2374-2381, 2017.

[8] S. Wan, Y. Xia, L. Qi, Y. H. Yang, and M. Atiquzzaman, "Automated colorization of a grayscale image with seed points propagation," IEEE Transactions on Multimedia, vol. 22, 2020.

[9] J. Lu, L. Ming, Z. Peng et al., "Remote-sensing image change detection with fusion of multiple wavelet kernels," IEEE Journal of Selected Topics in Applied Earth Observations \& Remote Sensing, vol. 9, no. 8, pp. 3405-3418, 2016.

[10] C. Shi, J. Zhang, H. Chen et al., "A novel hybrid method for remote sensing image approximation using the tetrolet transform," IEEE Journal of Selected Topics in Applied Earth Observations \& Remote Sensing, vol. 7, no. 12, pp. 4949-4959, 2017.

[11] C. Fan, L. Wang, P. Liu et al., "Compressed sensing based remote sensing image reconstruction via employing similarities of reference images," Multimedia Tools \& Applications, vol. 75, no. 19, pp. 1-25, 2016.

[12] M. Long, B. Du, C. He et al., "Region-of-Interest detection via superpixel-to-pixel saliency analysis for remote sensing image," IEEE Geoscience and Remote Sensing Letters, vol. 13, no. 12 , pp. 1-5, 2017.

[13] Xiang and H. Li, "Identification of remote sensing image of adverse geological body based on classification," Journal of 
Computational and Theoretical Nanoscience, vol. 13, no. 6, pp. 3790-3798, 2016.

[14] K. Liu, T. G. An, X. Huang et al., "Research on the difference between textures derived from DEM and remote-sensing image for topographic analysis," Journal of Geo-Information Science, vol. 18, no. 3, pp. 386-395, 2016.

[15] J. Munoz-Mari, E. Izquierdo-Verdiguier, M. Campos-Taberner et al., "HyperLabelMe: a web platform for benchmarking remote-sensing image classifiers," IEEE Geoscience and Remote Sensing Magazine, vol. 5, no. 4, pp. 79-85, 2017.

[16] T. Sheng and C. Qiang, "Dark channel prior-based altitude extraction method for a single mountain remote sensing image," IEEE Geoscience \& Remote Sensing Letters, vol. 14, no. 1, pp. 1-5, 2017.

[17] Q. Li, C. Yang, X. Liang et al., "Research on water information auto-extraction with object-oriented remote sensing imagetaking Tibet (East) region as an example," Journal of Geomatics, vol. 41, no. 6, pp. 40-43, 2016.

[18] J. Shen and J. I. Xuan, "Cloud and cloud shadow multi-feature collaborative detection from remote sensing image," Journal of Geo-Information Science, vol. 18, no. 5, pp. 599-605, 2016.

[19] Z. Y. Lv, H. He, B. Jón et al., "A generalized image scene decomposition-based system for supervised classification of very high resolution remote sensing imagery," Remote Sensing, vol. 8, no. 10, pp. 1-18, 2016.

[20] Q. Wang, Y. Li, and X. Liu, "The influence of photo elements on EEG signal recognition," Eurasip Journal on Image and Video Processing, vol. 2018, no. 1, p. 134, 2018.

[21] Z. Yang, L. Jie, and H. Min, "Remote sensing image transfer classification based on weighted extreme learning machine," IEEE Geoscience and Remote Sensing Letters, vol. 13, no. 10, pp. 1405-1409, 2016.

[22] E. Maggiori, Y. Tarabalka, G. Charpiat et al., "Convolutional neural networks for large-scale remote sensing image classification," IEEE Transactions on Geoscience \& Remote Sensing, vol. 55, no. 2, pp. 645-657, 2016.

[23] Y. Wang, L. Zhang, X. Tong et al., "A three-layered graphbased learning approach for remote sensing image retrieval," IEEE Transactions on Geoscience and Remote Sensing, vol. 54, no. 10 , pp. 1-15, 2016.

[24] Z. Du, X. Li, and X. Lu, "Local structure learning in high resolution remote sensing image retrieval," Neurocomputing, vol. 207, no. sep.26, pp. 813-822, 2016.

[25] X. Liu, Y. Li, and Q. Wang, "Multi-view hierarchical bidirectional recurrent neural network for depth video sequence based action recognition," International Journal of Pattern Recognition and Artificial Intelligence, vol. 32, no. 10, p. 1850033, 2018.

[26] Y. Li, Y. Zhang, H. Xin et al., "Large-scale remote sensing image retrieval by deep hashing neural networks," IEEE Transactions on Geoscience and Remote Sensing, vol. 56, no. 99, pp. 1-16, 2017.

[27] K. Sim, J. Yang, W. Lu, and X. Gao, "MaD-DLS: mean and deviation of deep and local similarity for image quality assessment," IEEE Transactions on Multimedia, no. 99, p. 1, 2020.

[28] J. Uthayakumar, M. Elhoseny, and K. Shankar, "Highly reliable and low-complexity image compression scheme using neighborhood correlation sequence algorithm in WSN," IEEE Transactions on Reliability, vol. 69, no. 4, pp. 1398-1423, 2020. 